\title{
A Case Study of Symptomatic Retroclival Ecchordosis Physaliphora: CT and MR Imaging
}

\author{
Craig Ferguson, David B. Clarke, Namita Sinha, Jai Jai Shiva Shankar
}

Key words: Ecchordosis, physaliphora, chordoma, CSF, leak, meningitis, diagnostic neuroradiology, neuroanatomy, meningitis, neuropathology, neurosurgery

doi:10.1017/cjn.2015.339

Can J Neurol Sci. 2016; 43: 210-212

Ecchordosis physaliphora (EP) is an uncommon notochordal remnant at the posterior clivus seen in approximately $2 \%$ of autopsies. Most of the patients are asymptomatic. It can be confused with its malignant counterpart, chordoma. ${ }^{1}$ We report a symptomatic middle-aged female with EP who presented with meningitis following a several-year history of intermittent cerebrospinal fluid (CSF) leak.

\section{Case Report}

A middle-aged patient presented to the emergency department with new onset of severe headache and confusion. The physical examination was remarkable for nuchal rigidity; the neurological examination showed no other focal findings. Laboratory testing revealed an elevated white blood count of $19.8 \times 10^{9} / \mathrm{L}$; a diagnosis of meningitis was confirmed after lumbar puncture revealed cloudy CSF with elevated white blood cells, high protein, and low glucose. The unenhanced computed tomography (CT) scan was unremarkable except for opacification of the sphenoid sinus with fluid. The patient was diagnosed with bacterial meningitis (Streptococcus salivarius grew from CSF samples) and treated with antibiotics. A subsequent CT scan of the paranasal sinuses performed 3 days later demonstrated a small clival defect associated with a small osseous stalk protruding into the prepontine cistern (Figure 1A). Magnetic resonance imaging done on the following day demonstrated a nonenhancing small T2-hyperintense and T1-hypointense retroclival lesion associated with a direct connection between the prepontine cistern and the sphenoid sinus suggestive of a CSF leak on the axial fast imaging employing steady-state acquisition sequence. A diagnosis of EP was suggested (Figure 1B-D).

The patient was treated for meningitis with a full recovery and continued to have CSF leakage. The patient was treated with an endoscopic endonasal approach for repair of sphenoid bony defect with fat graft after resection of prepontine lesion.

Microscopically, it consisted of sheets and clusters of large cells with central nucleus and vacuolated cytoplasm in myxomatous stroma, associated with fibrovascular tissue (Figure 2A, B). There was no significant mitotic activity (Ki67 labeling was less than 1\%) and no cellular pleomorphism. The characteristic physaliferous cells were immunoreactive to
S100 and monokeratin (Figure 2C, D, respectively), in keeping with ecchordosis physaliphora.

\section{DiscuSSION}

Most cases of EP are seen incidentally or on autopsy. Very few symptomatic cases of EP have been reported. ${ }^{2}$ Typically, symptoms are directly related to the mass effect of EP on the surrounding structures, perilesional hemorrhage, or CSF leak. ${ }^{1,2}$ There have been only four cases of EP associated with CSF leak reported in the literature. ${ }^{3-6} \mathrm{We}$ report the fifth case of EP presenting with CSF leak to remind readers that this can be a rare cause of meningitis.

$\mathrm{EP}$ is a benign, gelatinous hamartomatous lesion that arises from an ectopic notochordal remnant occurring along the midline craniospinal axis anywhere from the dorsum sella to the coccyx. ${ }^{1}$ It is found incidentally in approximately $0.5 \%$ to $2 \%$ of autopsies. ${ }^{2}$ Most of the intracranial EPs are found as retroclival masses located intradurally in the prepontine cistern. It is difficult to accurately differentiate between EP and its malignant counterpart chordoma $(2 \%-4 \%)$ clinically, radiologically, and even histopathologically. ${ }^{1,2}$ Distinction between EP and chordoma is important for treatment planning and prognosis, and there are certain characteristics that can be used to differentiate them.

CT scan has some role in diagnosing EP; it can typically demonstrate a tiny osseous stalk. ${ }^{1}$ Magnetic resonance imaging is the modality of choice in detecting, localizing, and characterizing EP. EP is typically T1-hypointense and T2-hyperintense. An important differentiating imaging feature of EP is the lack of gadolinium enhancement, whereas chordoma would show significant enhancement. ${ }^{1-3}$ Heavily T2-weighted image used for the CSF space is very useful in diagnosis of smaller lesions, such as that of our case. This sequence is also useful to delineate the CSF leakage. EP and

From the Faculty of Medicine and Dentistry, University of Alberta, Edmonton, Alberta, Canada (CF); Division of Neurosurgery, QE II Health Sciences Centre, Halifax, Nova Scotia, Canada (DBC); Department of Pathology, QE II Health Sciences Centre, Halifax, Nova Scotia, Canada (NS); Department of Diagnostic Radiology, QE II Health Sciences Centre, Halifax, Nova Scotia, Canada (JJSS).

Received December 15, 2014. Final Revisions Submitted July 30, 2015.

Correspondence to: Shankar, QE II Hospital, Radiology, Division of Neuroradiology, 1796 Summer Street, Halifax, Nova Scotia, Canada, B3H 3A6. Email: shivajai1@ gmail.com 

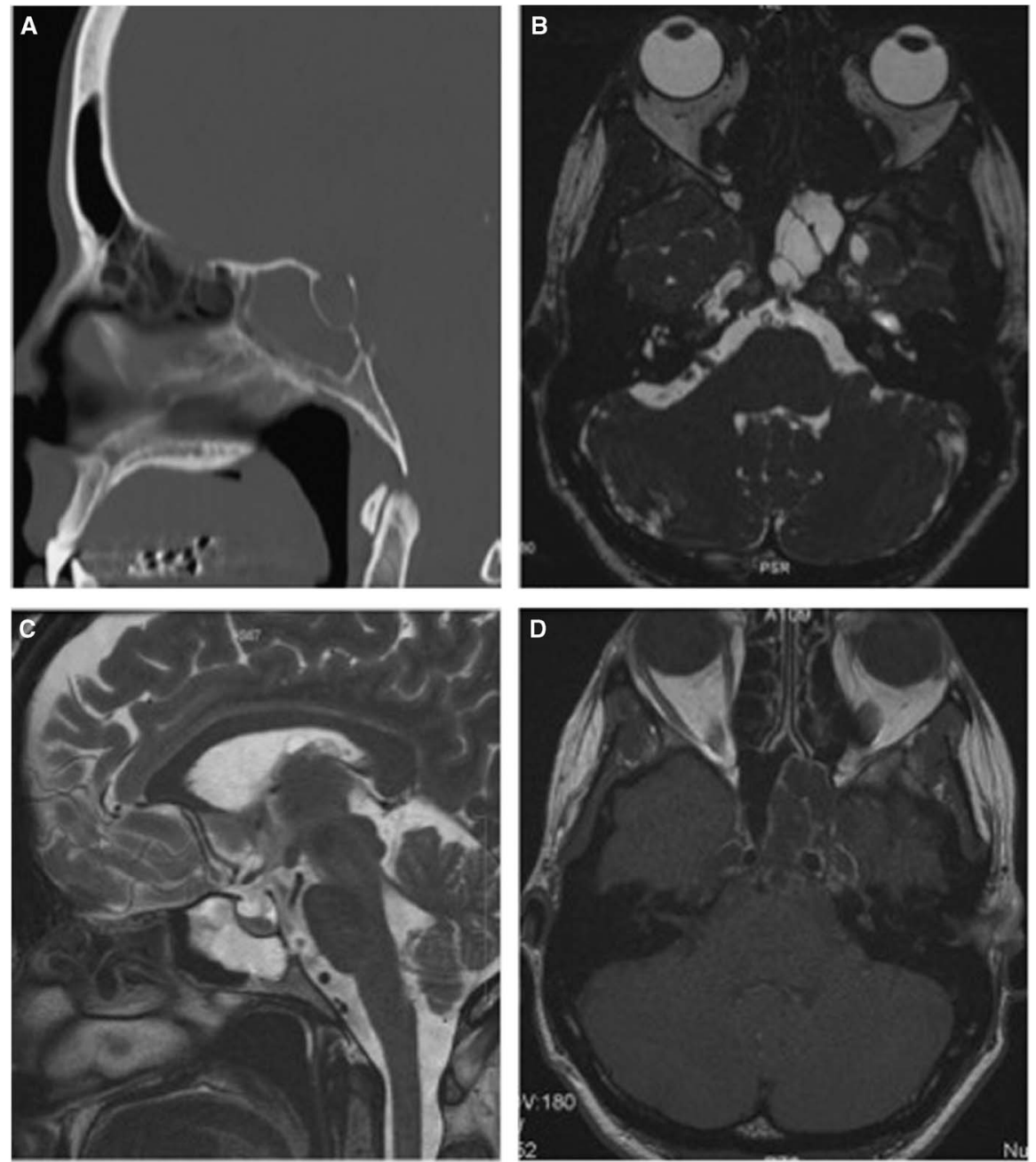

Figure 1: (A) Sinus computed tomography $(C T$; sagittal $3 \mathrm{~mm})$. There is a small retroclival defect associated with a small osseous stalk protruding into the prepontine cistern. Opacification of the sphenoid sinus with attenuation consistent with fluid suspicious of cerebrospinal fluid (CSF) leak. (B) Magnetic resonance imaging (MRI; 1.5 Tesla). Axial fast imaging using steadystate acquisition $(0.8 \mathrm{~mm}$ thickness). There is a small intermediate signal intensity lesion (straight arrow) attaching to the retroclivus via a short pedicle. There is a CSF leak (curve arrow) on the left side of the lesion from the prepontine cistern to the left sphenoid sinus. Dark signal intensity in the center of the lesion is the bony protrusion component as seen on prior CT. The findings are consistent of an ecchordosis physaliphora (EP) causing a CSF leak. (C) MRI (1.5 Tesla). Sagittal T2 (3-mm thickness). There is a small, mildly T2-hyperintense lesion attaching to the retroclivus via a short pedicle (arrow). There is an associated tiny bone defect in the midline clivus causing a CSF leak between the prepontine cistern and the left sphenoid sinus. The findings are consistent with an EP causing CSF leak. (D) MRI (1.5 Tesla). Postgadolinium axial T1 (2-mm thickness). There is no significant enhancement of the T1-hypointense retroclivus lesion, which favors a benign etiology such as EP rather than chordoma or metastasis. ${ }^{7,23}$

chordoma share a common embryonic notochordal origin, and therefore share similar histopathological features. Hypocellularity, sparse pleomorphism, and very low mitotic index favor the diagnosis of EP over chordoma, but are not pathognomonic. ${ }^{1}$

Five-year survival for chordoma is only about $10 \% .^{2}$ Clinically, most of the EPs are asymptomatic, whereas most previously reported cases of intradural chordoma had cranial nerve palsy or brain stem symptoms. ${ }^{1}$ The symptoms are directly related to the mass effect of EP on the surrounding structures, perilesional hemorrhage, or CSF leak. ${ }^{1,2}$ The cause of CSF leakage late in life in what is presumably a congenital lesion remains unknown; others have speculated that head injury, infection, or age-related bone density changes may be contributory. ${ }^{5}$ One might speculate that pulsations of the basilar artery contribute to a CSF leak; however, in our case, the dural defect was not immediately underneath the basilar artery.

To conclude, this case highlights the importance of identifying EP as a cause of CSF leak and meningitis. Following treatment of meningitis, surgery can be offered for removal of this benign entity and repair of the CSF fistula. 

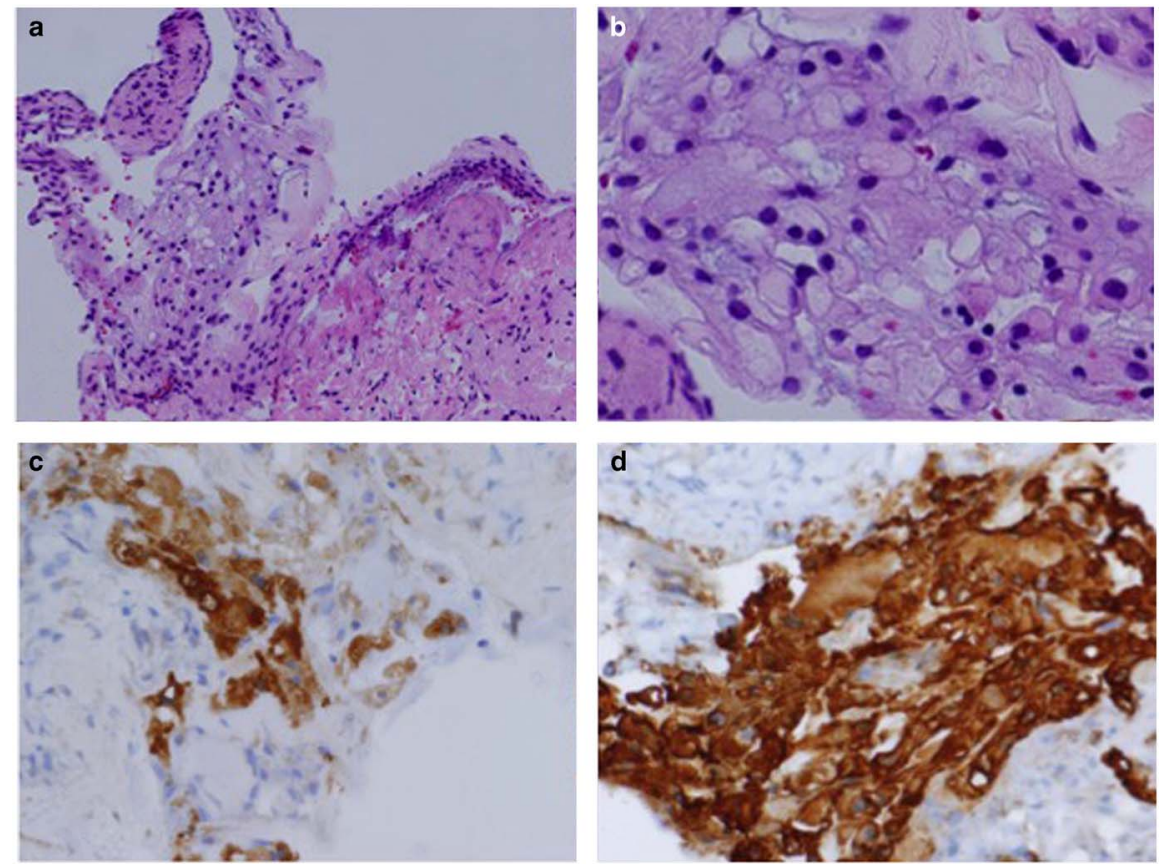

Figure 2: Biopsy from the mass shows a cluster of large physaliferous cells with a characteristic bubbly cytoplasm and a round to oval nuclei in the myxomatous stroma (A: hematoxylin and eosin, $\times 40 ;$ B: hematoxylin and eosin, $\times 200$ ). The lesional cells were immunoreactive to S100 $(C: \times 100)$ and monokeratin $(D: \times 100)$.

\section{ACKNOWLEDGEMENTS}

The authors thank Dr. Alex Easton for his assistance with preparing the histopathology.

Consent was not required for this study. There is no additional unpublished data from this study that will need to be made available. No funding was received for this project.

\section{Disclosures}

CF, DBC, NS, and JJSS have nothing to disclose.

\section{Statement OF AUTHORShIP}

JJSS and DC wrote the case report and searched the literature. This was JJSS's and DC's patient and they provided the leadership in starting this case. CF edited the report and provided an update on the literature search. NS provided the pathology for this case.

\section{REFERENCES}

1. Mehnert F, Beschorner R, Kuker W. Retroclival ecchordosis physaliphora: MR imaging and review of the literature. AJNR Am J Neuroradiol. 2004;25:1851-5.

2. Cha ST, Jarrahy R, Yong WH, Eby T, Shahinian HK. A rare symptomatic presentation of ecchordosis physaliphora and unique endoscope-assisted surgical management. Minim Invas Neurosurg. 2002:45:36-40.

3. Dias LA, Nakanishi M, Mangussi-Gomes J, Canuto M, Takano G, Oliveira CA. Successful endoscopic endonasal management of a transclival cerebrospinal fluid fistula secondary to ecchordosis physaliphora - an ectopic remnant of primitive notochord tissue in the clivus. Clin Neurol Neurosurg. 2014;117:116-9.

4. Bolzoni-Villaret A, Stefini R, Fontanella M, Bottazzoli M, Turri Zanoni M, Pistochini A, et al. Transnasal endoscopic resection of symptomatic ecchordosis physaliphora. Laryngoscope. 2014;124:1325-8.

5. Macdonald RL, Cusimano MD, Deck JH, Gullane PJ, Dolan EJ. Cerebrospinal fluid fistula secondary to Ecchordosis physaliphora. Neurosurgery. 1990;26:515-9.

6. Alli A, Clark M, Mansell NJ. Cerebrospinal fluid rhinorrhea secondary to ecchordosis physaliphora. Skull Base. 2008;18:395-9. 